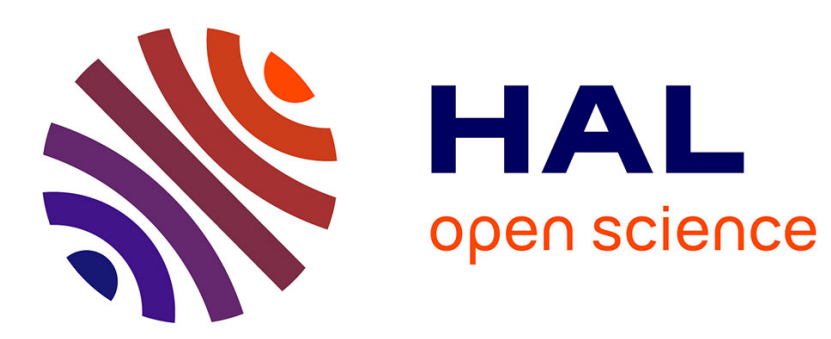

\title{
Delay Range Stability of distributed time delay systems
}

Frédéric Gouaisbaut, Yassine Ariba

\section{To cite this version:}

Frédéric Gouaisbaut, Yassine Ariba. Delay Range Stability of distributed time delay systems. 6th IFAC Symposium on Robust Control Design, Jun 2009, Haifa, Israel. 8p. hal-00357765

\section{HAL Id: hal-00357765 \\ https://hal.science/hal-00357765}

Submitted on 1 Feb 2009

HAL is a multi-disciplinary open access archive for the deposit and dissemination of scientific research documents, whether they are published or not. The documents may come from teaching and research institutions in France or abroad, or from public or private research centers.
L'archive ouverte pluridisciplinaire HAL, est destinée au dépôt et à la diffusion de documents scientifiques de niveau recherche, publiés ou non, émanant des établissements d'enseignement et de recherche français ou étrangers, des laboratoires publics ou privés. 


\title{
Delay Range Stability of distributed time delay systems
}

\author{
Frédéric Gouaisbaut*† and Yassine Ariba**
}

January 2008

\begin{abstract}
This paper is dedicated to the stability analysis of a class of uncertain distributed delay systems. We focus to the case where the kernel can be modelled as a polynomial function. The results are constructed by rewriting the system as an uncertain interconnected model and appropriate robust control tools as quadratic separation are then used to address to stability issue. Furthermore, some relations that highlight features of the delayed term are added to the interconnected model leading then to the conservatism reduction. At last, stability conditions are expressed in terms of LMIs which can be solved efficiently. Finally, some numerical examples show the effectiveness the proposed method.
\end{abstract}

\section{Introduction}

Since several decades, the problem of time delay stability have received a lot of attention (see the monographs by $[11,21]$ and references therein) since many dynamical processes can be described by functional differential equations including past values of the state. Distributed delay systems constitutes a particular case of such systems, modelling cumulative effect of the past values on the dynamics. Practical issues of such models are numerous in the literature. Distributed delay systems are often use to model the time lag phenomenon in thermodynamics (dynamics of combustion chambers in a liquid mono propellant rocket motor with pressure feeding, [5]), in ecology, epidemiology (predator-prey systems [8]). In all these cases, compared to a pointwise delay system, the use of a distributed kernel allows a thinner modelling of the interactions between the different system components. Nevertheless, establishing structural properties such as stability in the presence of the distributed kernel are much more difficult than the pointwise delay case. One first possibility is to use frequency approaches like [19] or [2] but these techniques are restricted to nominal systems without uncertainties. Another popular way to establish robust stability tests remains the use of adapted Lyapunov-Krasosvkii functional. However, in the literature, the main difficulty is generally avoided since a huge number of stability results has been devoted to a constant distributed kernel. Pioneer works $[16,15]$ use Lyapunov-Krasoskii functionals adapted to the distributed delay case. Extra terms are added to the classical Lyapunov Krasovskii to cope with

${ }^{*}$ Université de Toulouse; UPS, 118 Route de Narbonne, F-31062 Toulouse, France.

${ }^{\dagger}$ LAAS; CNRS; 7, avenue du Colonel Roche, F-31077 Toulouse, France. yariba@laas .fr 
the distributed delay term. It leads generally to Ricatti equations and are often conservative since the choice of Lyapunov-Krasovskii functional is very constrained. Another idea is to use a comparison principle [17, 23], which consists in replacing the original system by another one, simpler to study with a traditional Lyapunov-Krasovskii functional. The stability criterion is then expressed in terms of matrix measures and matrix norms. In that case, many variables have to be tuned which make the criterion difficult to handle. These techniques have been enhanced by $[6,7]$ and the descriptor method. The distributed delay system is then modelled as a singular system and the derivative of the state is explicitly taken into account for the construction of the Lyapunov functional. It leads to less conservative result which is expressed in terms of LMIs. These techniques have been recently refined by using either different bounding techniques (Moon or Jensen inequalities), [12],[3],[9] or some slack variables [18]. Nevertheless, all these results entail an inherent conservatism due to the constrained choice of the Lyapunov-krasovskii functional. An alternative proposed by $\mathrm{Gu}$ et al (see [11] and references therein) is to combine a very general class of Lyapunov Krasovskii functional proposed by Infante in [14] and a discretization scheme to obtain numerically tractable stability conditions. This technique dealing with piecewise constant delay kernel gives a drastic reduction of the conservatism at the expense of the numerical burden. Another interesting approach proposed by [20] dealing with a rational delay kernels relies on the use a a Lyapunov-Krasovskii approach and is combined with a full block S procedure to cope with the induced parametric LMI. Nevertheless, again stability is assessed only for a prescribed delay (pointwise delay stability) which does not provide robust criteria with respect to the delay.

In this paper, we adopt a different point of view to study the stability of such systems. First of all, we suppose that the distributed kernel is a polynomial function, a wider class of system compared to whose generally studied in the literature. Furthermore, in order to cope with such systems, we propose to use the quadratic separation approach. Coming from robust control theory, such tools study the robust stability of a linear transformation feedback interconnected with an uncertain transformation. This methodology have shown its efficiency to establish some stability conditions for linear time delay systems with a constant delay $[22,10]$ or time varying one [1]. Following the methodology proposed by [22], we model the distributed delay system as a nominal transformation connected to an uncertain operator composed of combinations of integral and some new delay distributed operators. Then, in order to reduce the conservatism, some relations between the uncertain operators and higher successive derivatives of the state are exploited to derive some new delay interval stability conditions. The paper is organised as follows. Next section is devoted to some preliminaries about the distributed delay system and quadratic separation. Then section 2 formulates a first way to describe the distributed delay system as an interconnected uncertain system. The following section gives the central results of the paper. Section 5 presents some numerical simulations. Notations : The set $\mathbb{L}_{2 e}^{n}$ is the extended set of $\mathbb{L}_{2}^{n}$ which consists of all measurable functions $f: \mathbb{R}^{+} \rightarrow \mathbb{C}^{n}$ such that the following norm $\|f\|_{2}=$ $\left(\int_{0}^{\infty}\left(f^{*}(t) f(t)\right)\right)^{1 / 2} d t<\infty .1_{\mathrm{p}}$ represents the $p \times p$ identity matrix. $0_{\mathrm{p} \times \mathrm{q}}$ stands 
for the $p \times q$ zero matrix. $\operatorname{diag}(A, B, C)$ stands for the block diagonal matrix:

$$
\operatorname{diag}(A, B, C)=\left[\begin{array}{ccc}
A & 0 & 0 \\
0 & B & 0 \\
0 & 0 & C
\end{array}\right] .
$$

\section{Problem statement}

In the sequel, we consider a continuous time distributed delay system of the form :

$$
\left\{\begin{array}{l}
\dot{x}(t)=A x(t)+\int_{-h}^{0} A_{d}(\theta) x(t+\theta) d \theta, \\
x(t)=\phi(t), \forall t \in\left[-h_{\max }, 0\right],
\end{array}\right.
$$

where $x(t) \in \mathbb{R}^{n}$ denotes the instantaneous state, the scalar $h>0$ is supposed to be unknown and belongs to the interval $\left[h_{\min }, h_{\max }\right], A \in \mathbb{R}^{n \times n} . \phi$ represents the initial conditions for system (1) and is a continuously differentiable function on $\left[-h_{\max }, 0\right]$. The function

$$
\begin{gathered}
A_{d}: \quad[-h, 0] \rightarrow \mathbb{R}^{n \times n}, \\
\theta \mapsto A_{d}(\theta),
\end{gathered}
$$

represents the distributed kernel of the delay system and in this paper, we focus on the case where the function $\theta \mapsto A_{d}(\theta)$ is a polynomial function of $\theta$ and can thus be written as:

$$
A_{d}(\theta)=\sum_{i=0}^{r} A_{d i} \theta^{i},
$$

where $r$ is a positive scalar corresponding to the polynomial order. $A_{d i} \in$ $\mathbb{R}^{n \times n}, \forall i \in\{0, \ldots, r\}$ are real constant matrices. Hence, the distributed delay system (1) can be reformulated as:

$$
\dot{x}(t)=A x(t)+\sum_{i=0}^{r} A_{d i} \int_{-h}^{0} \theta^{i} x(t+\theta) d \theta .
$$

Remark 1 Taking the distributed kernel as a polynomial function is not so restrictive since every sufficiently smooth function on a compact interval $[-h, 0]$ can be approximated by a polynomial function.

In this paper, we aim at finding LMI conditions which ensures the stability of (2) for all delays in an interval $\left[h_{\min }, h_{\max }\right]$ where $h_{\min }$ is possibly set to zero if delay dependent stability tests are looked for. To this end, we propose to use the quadratic separation framework developed by [13] and extended to the singular case by [22]. This latter methodology, inherited from the robust analysis is recalled in the following. Consider the feedback connection depicted in Figure 1, where:

- $\mathcal{E}$ and $\mathcal{A}$ are two possibly non-square matrices;

- $\nabla$ is an uncertain constant, complex valued matrix, with appropriate dimensions, that belongs to some set $\mathbb{W}$. 


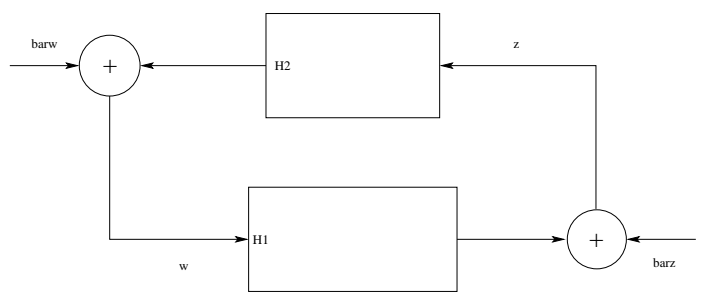

Figure 1: Feedback system

For simplicity of calculus, we will suppose that $\mathcal{E}$ is full column rank. We make no assumption on the uncertainty set $\mathbb{W}$.

The following theorem provides a condition for the well-posedness of the interconnection, as well as its stability.

Theorem 1 [22] The uncertain feedback system of Figure 1 is well-posed and stable if and only if there exists a Hermitian matrix $\Theta=\Theta^{*}$ satisfying both conditions

$$
\begin{gathered}
{\left[\begin{array}{cc}
\mathcal{E} & -\mathcal{A}
\end{array}\right]^{\perp *} \Theta\left[\begin{array}{ll}
\mathcal{E} & -\mathcal{A}
\end{array}\right]^{\perp}>0} \\
{\left[\begin{array}{c}
I \\
\nabla
\end{array}\right]^{*} \Theta\left[\begin{array}{c}
I \\
\nabla
\end{array}\right] \leq 0 \quad, \quad \forall \nabla \in \mathbb{W} .}
\end{gathered}
$$

If $\mathcal{E}$ and $\mathcal{A}$ are real matrices, the equivalence still holds with $\Theta$ restricted to be a real matrix.

The next section shows how to formulate the distributed delay system (2) as an interconnected feedback system of the form of Figure (1) in order to use Theorem 2.

\section{Preliminary results}

Fist of all, define the integral operator $\mathcal{I}$ and the distributed delay operator $\forall i \in\{1, \ldots, r\}, \delta_{i}$ by

$$
\begin{aligned}
\delta_{i}: \mathbb{L}_{2 e} & \rightarrow \mathbb{L}_{2 e}, \\
x(t) & \rightarrow \int_{-h}^{0} \theta^{i} x(t+\theta) d \theta
\end{aligned}
$$

and

$$
\begin{aligned}
\mathcal{I}: \quad \mathbb{L}_{2 e} & \rightarrow \mathbb{L}_{2 e}, \\
x(t) & \rightarrow \int_{0}^{t} x(\theta) d \theta .
\end{aligned}
$$

Hence, using $\mathcal{I}$ and $\delta_{i}$, the distributed delay system (2) is recast as a feedback interconnection as Figure (1) with

$$
\underbrace{\left[\begin{array}{c}
x(t) \\
\left(1_{\mathrm{n}} \delta_{0}\right)[\mathrm{x}(\mathrm{t})] \\
\vdots \\
\left(1_{\mathrm{n}} \delta_{\mathrm{r}}\right)[\mathrm{x}(\mathrm{t})]
\end{array}\right]}_{w(t)}=\underbrace{\left[\begin{array}{cccc}
\mathcal{I} 1_{\mathrm{n}} & & & 0 \\
& \delta_{0} 1_{\mathrm{n}} & & \\
& & \ddots & \\
0 & & & \delta_{r} 1_{\mathrm{n}}
\end{array}\right]}_{\nabla} \underbrace{\left[\begin{array}{c}
\dot{x}(t) \\
x(t) \\
\vdots \\
x(t)
\end{array}\right]}_{z(t)},
$$


and

$$
\left[\begin{array}{c}
\dot{x}(t) \\
x(t) \\
\vdots \\
x(t)
\end{array}\right]=\underbrace{\left[\begin{array}{cccc}
A & A_{d 0} & \ldots & A_{d r} \\
1 & 0 & \ldots & 0 \\
\vdots & \vdots & & \vdots \\
\mathrm{I} & 0 & \ldots & 0
\end{array}\right]}_{\mathcal{A}}\left[\begin{array}{c}
x(t) \\
\left(1_{\mathrm{n}} \delta_{0}\right)[\mathrm{x}(\mathrm{t})] \\
\vdots \\
\left(1_{\mathrm{n}} \delta_{\mathrm{r}}\right)[\mathrm{x}(\mathrm{t})]
\end{array}\right]
$$

Note that since $h$ is an unknown constant, the operators $\delta_{i}$ can be conveniently expressed in the Laplace domain by $\delta_{i}(s)=\int_{-h}^{0} \theta^{i} e^{\theta s} d \theta$. Following [13], the uncertainty set $\mathbb{W}$ and $\nabla$ get then a more simple form:

$$
\mathbb{W}=\left\{\nabla(s), \forall s \in \mathbb{C}^{+}\right\}
$$

with

$$
\nabla(s)=\operatorname{diag}\left(s^{-1} 1_{\mathrm{n}}, \delta_{0}(\mathrm{~s}) 1_{\mathrm{n}}, \ldots, \delta_{\mathrm{r}}(\mathrm{s}) 1_{\mathrm{n}}\right) .
$$

This last formulation of $\mathbb{\mathbb { V }}$ allow then to construct some parameterized separators $\Theta$ satisfying the second inequality (4) as stated by the following Lemma.

Lemma 1 Consider the uncertain operator $\nabla$ given by (10), a separator $\Theta$ satisfying (4) is given by

$$
\Theta=\left[\begin{array}{cc}
\Theta_{11} & \Theta_{12} \\
\Theta_{12}^{*} & \Theta_{22}
\end{array}\right]
$$

with

$$
\begin{aligned}
& \Theta_{11}=\operatorname{diag}\left(0,-h_{\max }^{2} Q_{0}, \ldots,-\frac{h_{\text {max }}^{2 r+2}}{(r+1)^{2}} Q_{r}\right), \\
& \Theta_{12}=\operatorname{diag}(-P, 0, \ldots, 0), \\
& \Theta_{22}=\operatorname{diag}\left(0, Q_{0}, \ldots, Q_{r}\right),
\end{aligned}
$$

and $P, Q_{0}, \ldots, Q_{r} \in \mathbb{R}^{n \times n}$ are positive definite matrices.

Proof 1 The key idea is to construct separators for each uncertainties $s^{-1}, \delta_{0}, \ldots \ldots, \delta_{r}$ which compose $\nabla$ and finally concatenate all these relations to construct the whole separator $\Theta$. Noting that $\forall s \in \mathbb{C}^{+}$,

$$
\left|\delta_{i}(s)\right| \leq \int_{-h}^{n}\left|\theta^{i} e^{\theta s}\right| d \theta \leq \frac{h_{\max }^{i+1}}{i+1},
$$

and following [10], a first set of separators for $\delta_{i}$, parameterized by $Q_{i}>0$ can be defined using

$$
\left[\begin{array}{c}
1_{\mathrm{n}} \\
\delta_{i} 1_{\mathrm{n}}
\end{array}\right]^{*}\left[\begin{array}{cc}
-\frac{h_{\max }^{2 i+2)}}{(i+1)^{2}} Q_{i} & 0 \\
0 & Q_{i}
\end{array}\right]\left[\begin{array}{c}
1_{\mathrm{n}} \\
\delta_{i} 1_{\mathrm{n}}
\end{array}\right]<0
$$

Furthermore, following [22], the separator for $\mathcal{I}$ is parameterized by a positive definite matrix $P$ which the following inequality:

$$
\left[\begin{array}{c}
1_{\mathrm{n}} \\
s^{-1} 1_{\mathrm{n}}
\end{array}\right]^{*}\left[\begin{array}{cc}
0 & -P \\
-P & 0
\end{array}\right]\left[\begin{array}{c}
1_{\mathrm{n}} \\
s^{-1} 1_{\mathrm{n}}
\end{array}\right]<0 .
$$

Consequently, gathering all these inequalities, the separator related to $\nabla$ defined by (7) can be chosen as (11). 
At this stage, combining this last lemma with Theorem (2), we state the first delay dependent stability result :

Theorem 2 For given positive scalars $h_{\max }$ and $r$, if there exists positive definite matrices $P, Q_{i}$ for $i=\{0, \ldots, r\} \in \mathbb{R}^{n \times n}$, then system (2) is asymptotically stable $\forall h \leq h_{\max }$ if the LMI condition (3) holds with $\Theta, \mathcal{E}$ and $\mathcal{A}$ defined as (11) and (7), (8).

Proof 2 In order to prove this theorem, we first model the original system (2) into an interconnected system (7-8). Then, applying Theorem 1, since Lemma 1 fulfils the second requirement (4), the first inequality (3) gives the condition to be tested.

\section{Main Results}

The proposed idea is to introduce new relevant relations between the different components of the distributed delay system. The previous result can be improved by getting some new relations between $\delta_{i}$ and $\delta_{i-1}$ summarised by the following lemma.

Lemma 2 Given $i \geq 1$,

$$
\begin{aligned}
\delta_{i}(s) & =-\frac{(-h)^{i} e^{-s h}}{s}-\frac{i}{s} \delta_{i-1}(s), \\
\text { with } \delta_{0}(s) & =\frac{1-e^{-h s}}{s} .
\end{aligned}
$$

Proof 3 Integrating by part $\delta_{i}(s)=\int_{-h}^{0} \theta^{i} e^{\theta s} d \theta$, we get :

$$
\delta_{i}(s)=-\frac{(-h)^{i} e^{-s h}}{s}-\frac{i}{s} \int_{-h}^{0} \theta^{i-1} e^{s \theta} d \theta,
$$

which readily leads to the proposed relation.

This new relation links three different operators $\delta_{i}, \delta_{i-1}$ and $s^{-1}$ and the delay operator defined by

$$
\begin{aligned}
\mathcal{D}: & \mathbb{L}_{2 e} \rightarrow \mathbb{L}_{2 e}, \\
& x(t) \rightarrow x(t-h) .
\end{aligned}
$$

and expressed using Laplace transform by $e^{-h s}$. Applying theses relations to the system instantaneous state $x(t)$ give us a new set of relations between the state $x(t)$ and the delayed state $x(t-h)$ :

$$
\left\{\begin{array}{l}
\left(1_{\mathrm{n}} \delta_{0}\right)[\dot{\mathrm{x}}(\mathrm{t})]=\mathrm{x}(\mathrm{t})-\mathrm{x}(\mathrm{t}-\mathrm{h}) \\
\left(1_{\mathrm{n}} \delta_{1}\right)[\dot{\mathrm{x}}(\mathrm{t})]=\mathrm{hx}(\mathrm{t}-\mathrm{h})-\delta_{0}[\mathrm{x}(\mathrm{t})] \\
\left(1_{\mathrm{n}} \delta_{2}\right)[\dot{\mathrm{x}}(\mathrm{t})]=-\mathrm{h}^{2} \mathrm{x}(\mathrm{t}-\mathrm{h})-2 \delta_{1}[\mathrm{x}(\mathrm{t})] \\
\vdots \\
\left(1_{\mathrm{n}} \delta_{\mathrm{r}}\right)[\dot{\mathrm{x}}(\mathrm{t})]=-(-\mathrm{h})^{\mathrm{r}} \mathrm{x}(\mathrm{t}-\mathrm{h})-\mathrm{r} \delta_{\mathrm{r}-1}[\mathrm{x}(\mathrm{t})]
\end{array}\right.
$$


As it appears that the derivative of $x(t)$ plays a central role in these relations, we thus propose to consider the original model (2) as well as its derivative to obtain a new model:

$$
\left\{\begin{array}{l}
\dot{x}(t)=A x(t)+\sum_{i=0}^{r} A_{d i} \int_{-h}^{0} \theta^{i} x(t+\theta) d \theta \\
\ddot{x}(t)=A \dot{x}(t)+\sum_{i=0}^{r} A_{d i} \int_{-h}^{0} \theta^{i} \dot{x}(t+\theta) d \theta
\end{array}\right.
$$

In order to take into account all these new relations (15) and (16), we choose to model system (16) by a new interconnected system with an uncertain transformation given by

$$
\mathbb{W}=\left\{\nabla(s), \forall s \in \mathbb{C}^{+}\right\}
$$

with

$$
\nabla(s)=\operatorname{diag}\left(\begin{array}{l}
1_{2 \mathrm{n}} \mathrm{s}^{-1}, 1_{\mathrm{n}} \mathrm{e}^{-\mathrm{hs}}, \\
\left.1_{2 \mathrm{n}} \delta_{0}(\mathrm{~s}), \ldots, 1_{2 \mathrm{n}} \delta_{\mathrm{r}}(\mathrm{s})\right),
\end{array}\right.
$$

At this stage, following the methodology proposed in the last section, we construct a separator $\Theta$ for the uncertain set described by (17).

Lemma 3 Consider the uncertain operator $\nabla$ given by (18), a separator $\Theta$ satisfying (4) is given by

$$
\Theta=\left[\begin{array}{l|l}
\Theta_{11} & \Theta_{12} \\
\hline \Theta_{12}^{T} & \Theta_{22}
\end{array}\right],
$$

where

$$
\begin{aligned}
& \Theta_{11}=\operatorname{diag}\left(0,-\mathrm{R},-\mathrm{h}_{\max }^{2} \mathrm{Q}_{0}, \ldots,-\frac{-\mathrm{h}_{\text {max }}^{2 r+2}}{(\mathrm{r}+1)^{2}} \mathrm{Q}_{\mathrm{r}}\right), \\
& \Theta_{12}=\operatorname{diag}(-P, 0, \ldots, 0), \\
& \Theta_{22}=\operatorname{diag}\left(0, \mathrm{R}, \mathrm{Q}_{0}, \ldots, \mathrm{Q}_{\mathrm{r}}\right),
\end{aligned}
$$

with $P, Q_{0}, \ldots, Q_{r} \in \mathbb{R}^{2 n \times 2 n} R \in \mathbb{R}^{n \times n}, r+3$ positive definite matrices.

Proof 4 This proof follows essentially the same idea than the one of Lemma 1, except that an additional uncertainty $e^{-h s}$, representing the pointwise delay is added to $\nabla$. Noting that $\forall s \in \mathbb{C}^{+},\left|e^{-h s}\right| \leq 1$, we get

$$
\left[\begin{array}{c}
1_{\mathrm{n}} \\
e^{-h s} 1_{\mathrm{n}}
\end{array}\right]^{*}\left[\begin{array}{cc}
-R & 0 \\
0 & R
\end{array}\right]\left[\begin{array}{c}
1_{\mathrm{n}} \\
e^{-h s} 1_{\mathrm{n}}
\end{array}\right]<0 .
$$

Gathering the former relations (12), (13), it is straightforward to prove that separator (19) along with (18) satisfies inequality (4), which concludes the proof.

Having chosen the uncertainty $\nabla(17)$ to model system (2) as an interconnected system of Figure (1), we define the matrices as well as the internals signals which compose the linear transformation $\mathcal{E}(z-\bar{z})=\mathcal{A} \omega$ as:

$$
z(t)=\left[\begin{array}{c}
\ddot{x}(t) \\
\dot{x}(t) \\
x(t) \\
\hline \dot{x}(t) \\
x(t) \\
\hline \vdots \\
\hline \dot{x}(t) \\
x(t)
\end{array}\right], w(t)=\left[\begin{array}{c}
\dot{x}(t) \\
x(t) \\
x(t-h) \\
\left.\hline\left(1_{\mathrm{n}} \delta_{0}\right)[\dot{\mathrm{x}} \mathrm{t})\right] \\
\frac{\left(1_{\mathrm{n}} \delta_{0}\right)[\mathrm{x}(\mathrm{t})]}{\vdots} \\
\frac{\vdots}{\left(1_{\mathrm{n}} \delta_{\mathrm{r}}\right)[\dot{\mathrm{x}}(\mathrm{t})]} \\
\left(1_{\mathrm{n}} \delta_{\mathrm{r}}\right)[\mathrm{x}(\mathrm{t})]
\end{array}\right] .
$$


Defining $\forall i \geq 0, \bar{A}_{d_{i}}=1_{2} \otimes \mathrm{A}_{\mathrm{d}_{\mathrm{i}}}$,

$$
\bar{A}=\left[\begin{array}{ccc}
A & 0 & 0 \\
0 & A & 0
\end{array}\right], F_{1}=\left[\begin{array}{ccc}
1 & 0 & 0 \\
0 & 1 & 0 \\
0 & 1 & 0 \\
0 & 0 & 1
\end{array}\right], V=\left[\begin{array}{c}
1 \\
\vdots \\
1
\end{array}\right] \in \mathbb{R}^{(r+2)},
$$

and

$$
A_{L}=\left[\begin{array}{ccccccccc}
0 & 1 & -1 & -1 & 0 & \ldots \ldots \ldots \ldots & 0 \\
0 & 0 & h 1 & 0 & -1 & -1 & 0 & \ldots & 0 \\
\vdots & \vdots & \vdots & \vdots & \ddots & \ddots & \ddots & \ddots & \vdots \\
0 & 0 & -(-h)^{r} 1 & 0 & \ldots & 0 & -r 1 & -1 & 0
\end{array}\right]
$$

The matrices defining the linear transformation are then given by :

$$
\begin{aligned}
& \mathcal{E}=\left[\begin{array}{c}
E_{H} \\
0_{\mathrm{n}(\mathrm{r}+1) \times \mathrm{n}(2 \mathrm{r}+5)}
\end{array}\right], E_{H}=\operatorname{diag}\left(F_{1}, 1_{2 \mathrm{n}(\mathrm{r}+1)}\right), \\
& \mathcal{A}=\left[\begin{array}{cccc}
\bar{A} & \bar{A}_{d 0} & \ldots & \bar{A}_{d r} \\
\hline \mathrm{V} \otimes 1_{2 \mathrm{n}} & & & 0_{2 \mathrm{n}(\mathrm{r}+2) \times \mathrm{n}(2 \mathrm{r}+3)}
\end{array}\right],
\end{aligned}
$$

At this stage, we propose a second theorem:

Theorem 3 For given positive scalars $h, h_{\max }, r$ such that $0 \leq h \leq h_{\max }$, if there exists positive definite matrices $P, Q_{0}, \ldots, Q_{r} \in \mathbb{R}^{2 n \times 2 n}$ and $R \in \mathbb{R}^{n \times n}$, then system (2) is asymptotically stable for the given $h$, if the LMI condition (3) holds with $\Theta, \mathcal{E}$ and $\mathcal{A}$ defined as (19) and (22).

Proof 5 This proof is essentially the same than the proof of theorem 2 and is thus omitted.

It is worth to note that this result proves that system (2) is only stable for a prescribed $h$ such that $h \leq h_{\max }$ and not $\forall h \leq h_{\max }$. This drawback can be eliminating by combining these two expressions:

$$
\left\{\begin{array}{l}
\delta_{i}(s)=-\frac{(-h)^{i} e^{-s h}}{s}-\frac{i}{s} \delta_{i-1}(s), \\
\delta_{i-1}(s)=-\frac{(-h)^{i-1} e^{-s h}}{s}-\frac{i-1}{s} \delta_{i-2}(s) .
\end{array}\right.
$$

It results that

$$
\delta_{i}(s)=-\left(h+\frac{i}{s}\right) \delta_{i-1}(s)-\frac{h(i-1)}{s} \delta_{i-2}(s), \forall i \geq 2,
$$

This last equation proves that the relation between $\delta_{i}, \delta_{i-1}, \delta_{i-2}$ is linear with respect to $h$.

Furthermore, some calculations show that:

$$
\delta_{i}(s)=(-1)^{i} i !\left(\frac{1}{s^{i+1}}-\sum_{j=0}^{i} \frac{h^{i-j} e^{-s h}}{(i-j) ! s^{j+1}}\right)
$$

This last expression shows that the uncertainty $\delta_{i}(s)$ can be described on the combination of $e^{-h s}$ and integrals up to powers $i+1$. Hence, in order to take 
into advantage of these new expressions, following the work of [4], we consider the augmented vector:

$$
y(t)=\left[\begin{array}{c}
x^{l}(t) \\
x^{l-1}(t) \\
\vdots \\
\dot{x}(t) \\
x(t)
\end{array}\right] .
$$

This extended vector and its derivative are linked by the linear function:

$$
\underbrace{\left[\begin{array}{cccc}
0 & 1 & & \\
\vdots & & \ddots & \\
0 & & & 1
\end{array}\right]}_{E_{2}} \dot{y}(t)=\underbrace{\left[\begin{array}{cccc}
1 & & & 0 \\
& \ddots & & \vdots \\
& & 1 & 0
\end{array}\right]}_{E_{1}} y(t)
$$

We propose then to use the following uncertain set:

$$
\mathbb{W}=\left\{\nabla(s), \forall s \in \mathbb{C}^{+}\right\},
$$

with

$$
\nabla(s)=\operatorname{diag}\left(\begin{array}{ll} 
& 1_{\mathrm{n}(1+1)} \mathrm{s}^{-1}, 1_{\mathrm{n}(1+1)} \mathrm{e}^{-\mathrm{hs}}, \\
& \left.1_{2 \mathrm{n}(1+1)} \delta_{0}(\mathrm{~s}), \ldots, 1_{2 \mathrm{n}(1+1)} \delta_{\mathrm{r}}(\mathrm{s})\right)
\end{array}\right.
$$

Lemma 4 Consider the uncertain operator $\mathbb{W}$ given by (25), a separator $\Theta$ satisfying (4) is of the form (19) with $R, P \in \mathbb{R}^{n(l+1) \times n(l+1)}, Q_{0}, \ldots, Q_{r} \in$ $\mathbb{R}^{2 n(l+1) \times 2 n(l+1)}$ some positive definite matrices.

\section{Proof 6 Omitted.}

Choosing the uncertain transformation $\nabla$ by (25), the modelling of system (16) is described as follows. Firstly, we introduce the internal signals:

$$
z(t)=\left[\begin{array}{c}
\dot{y}(t) \\
y(t) \\
\hline \dot{y}(t) \\
y(t) \\
\hline \vdots \\
\hline \dot{y}(t) \\
y(t)
\end{array}\right], w(t)=\left[\begin{array}{c}
y(t) \\
\frac{y(t-h)}{\left(1_{\mathrm{n}(1+1)} \delta_{0}\right)[\dot{y}(\mathrm{t})]} \\
\frac{\left(1_{\mathrm{n}(1+1)} \delta_{0}\right)[\mathrm{y}(\mathrm{t})]}{\vdots} \\
\frac{\vdots}{\left(1_{\mathrm{n}(1+1)} \delta_{\mathrm{r}}\right)[\dot{\mathrm{y}}(\mathrm{t})]} \\
\left(1_{\mathrm{n}(1+1)} \delta_{\mathrm{r}}\right)[\mathrm{y}(\mathrm{t})]
\end{array}\right] .
$$

Then, we define some intermediate matrices,

$$
W=\left[\begin{array}{cccc}
1 & 0 & \ldots & 0 \\
-1 & 1 & \ldots & 0 \\
\vdots & & \ddots & \vdots \\
-1 & & \ldots & 1
\end{array}\right] \in \mathbb{R}^{(r+2) \times(r+2)},
$$




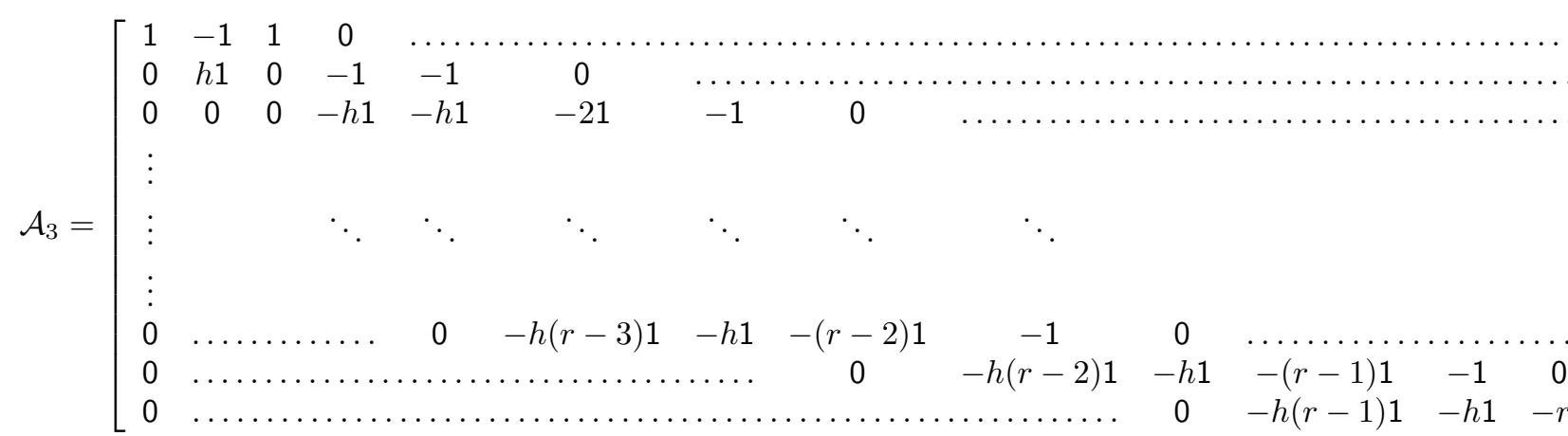

(28)

$$
\begin{aligned}
& \mathcal{E}_{1}=W \otimes 1_{2 \mathrm{n}(1+1)} \in \mathbb{R}^{(2 \mathrm{n}(\mathrm{r}+2)(1+1)) \times(2 \mathrm{n}(\mathrm{r}+2)(1+1))}, \\
& \mathcal{E}_{2}=\left[\begin{array}{lllll}
E_{2} & -E_{1} & 0 & \ldots & 0,
\end{array}\right] \in \mathbb{R}^{n l \times 2 n(r+2)(l+1)}, \\
& \mathcal{E}_{3}=0_{\mathrm{n}(1+1)(\mathrm{r}+1) \times 2 \mathrm{n}(1+1)(\mathrm{r}+2)}, \\
& \mathcal{E}_{4}=0_{\mathrm{nl}(\mathrm{r}+1) \times 2 \mathrm{n}(1+1)(\mathrm{r}+2),}
\end{aligned}
$$

which allow to define the matrix $\mathcal{E}$ :

$$
\mathcal{E}=\left[\begin{array}{l}
\mathcal{E}_{1} \\
\hline \mathcal{E}_{2} \\
\hline \mathcal{E}_{3} \\
\hline \mathcal{E}_{4}
\end{array}\right]
$$

In order to define the matrix $\mathcal{A}$, let introduce

$$
\begin{aligned}
\bar{A} & =1_{1+1} \otimes \mathrm{A}, \overline{\mathrm{A}}_{\mathrm{di}}=1_{\mathrm{l}+1} \otimes \mathrm{A}_{\mathrm{di}}, \forall \mathrm{i} \geq 0, \\
\mathcal{A}_{1} & =\left[\begin{array}{cccccccc}
\bar{A} & 0 & 0 & \bar{A}_{d 0} & 0 & \ldots & 0 & \bar{A}_{d r} \\
1_{(1+1) \mathrm{n}} & 0 & \ldots \ldots \ldots \ldots \ldots \ldots & 0
\end{array}\right], \\
\mathcal{A}_{2} & =0_{(2 \mathrm{n}(\mathrm{r}+2)(1+1)) \times(2 \mathrm{n}(\mathrm{r}+1)(1+1))}, \\
\mathcal{A}_{4} & =\left[\begin{array}{lll}
0_{(\mathrm{r}+1) \times 1} & 1_{\mathrm{r}+1}
\end{array}\right] \otimes\left[\begin{array}{ll}
E_{2} & -E_{1}
\end{array}\right],
\end{aligned}
$$

These matrices $\mathcal{A}_{1}, \mathcal{A}_{2}, \mathcal{A}_{3}, \mathcal{A}_{4}$ allow then to construct the matrix $\mathcal{A}(h)$, which depends linearly of $h$ :

$$
\mathcal{A}(h)=\left[\begin{array}{l}
\mathcal{A}_{1} \\
\mathcal{A}_{2} \\
\hline \mathcal{A}_{3} \\
\hline \mathcal{A}_{4}
\end{array}\right] .
$$


Now, a delay-range dependent stability result can be developed:

Theorem 4 For given positive scalars $h_{\min }, h_{\max }, r, l$ such that $h_{\min } \leq h_{\max }$, if there exists positive definite matrices $Q_{0}, \ldots, Q_{r} \in \mathbb{R}^{2 n(l+1) \times 2 n(l+1)}, P, R \in$ $\mathbb{R}^{n(l+1) \times n(l+1)}$ and a matrix $X \in \mathbb{R}^{2 n(l+1)(5+2 r) \times(n l(4 r+7)+n(3 r+5))}$, such that

$$
\left\{\begin{array}{c}
\Theta+X S\left(h_{\min }\right)+S\left(h_{\min }\right)^{T} X^{T}>0 \\
\Theta+X S\left(h_{\max }\right)+S\left(h_{\max }\right)^{T} X^{T}>0
\end{array}\right.
$$

with

$$
\begin{aligned}
& S\left(h_{\min }\right)=\left[\begin{array}{ll}
\mathcal{E} & -\mathcal{A}\left(h_{\min }\right)
\end{array}\right], \\
& S\left(h_{\max }\right)=\left[\begin{array}{ll}
\mathcal{E} & -\mathcal{A}\left(h_{\max }\right)
\end{array}\right],
\end{aligned}
$$

and $\Theta, \mathcal{E}, \mathcal{A}(h)$ defined as (19) and (27),(29), then system (2) is asymptotically stable for $h$, such that $h_{\min } \leq h \leq h_{\max }$.

Proof 7 The proof follows the same lines than the one of Theorem 3. We use Theorem 2 to the uncertain interconnection defined by equations (25) and (27),(29). By construction of the separator (19), the second requirement (4) is fulfilled. Then, the first requirement (3) gives that

$$
\left[\begin{array}{ll}
\mathcal{E} & -\mathcal{A}(h)
\end{array}\right]^{\perp *} \Theta\left[\begin{array}{ll}
\mathcal{E} & -\mathcal{A}(h)
\end{array}\right]^{\perp}>0 .
$$

This last inequality is not linear in $h$ but using Finsler Lemma, a sufficient condition for (31) is :

$$
\Theta+X\left[\begin{array}{ll}
\mathcal{E} & -\mathcal{A}(h)
\end{array}\right]+\left[\begin{array}{ll}
\mathcal{E} & -\mathcal{A}(h)
\end{array}\right]^{T} X^{T}>0,
$$

with $X$ a matrix of appropriate dimensions. This last inequality is then linear in $h$ and this inequality has to be assessed on the 2 vertices of the polytop generated by the interval on $h$, which concludes the proof.

Remark 2 The system (2) is modeled in a robust framework, the extension of previous result to the robust case is straightforward and thus will not be explained.

\section{$5 \quad$ Examples}

In this section, two numerical examples are provided to show the effectiveness of the proposed methodology. The first example is given by:

$$
\dot{x}(t)=-2 x(t)+\int_{-h}^{0}\left(1+\theta+\theta^{3}\right) x(t+\theta) d \theta .
$$

Using an analytical method proposed by [2], system (33) is shown to be asymptotically stable for all delays less than 1.759 . Using theorem (3), we prove that system (33) is asymptotically stable $\forall h<1.0$. Then, choosing $h_{\min }=0$ and using theorem (4), we get the following results summarized in table 1.

In that example, using an augmented vector to model the original system allows a reduction of conservatism. Nevertheless, for $l \geq 3$, increasing $l$ do not improve anything. Moreover, surprisingly, if the kernel $A_{d}(\theta)=1+\theta+\theta^{3}$ is modeled as a polynomial of order greater than $r$, taking the distributed kernel as 


\begin{tabular}{|c|c|}
\hline Method & $h_{\max }$ \\
\hline Theorem $4, l=0$ & 1.32 \\
Theorem $4, l=1$ & 1.58 \\
Theorem $4, l=2$ & 1.60 \\
Theorem $4, l \geq 3$ & 1.61 \\
\hline
\end{tabular}

Table 1: $h_{\max }$ for system (33) w.r.t. $l$

\begin{tabular}{|c|c|}
\hline Theorem $4, l=0$ & $h_{\max }$ \\
\hline$r=3$ & 1.32 \\
$r=4$ & 1.43 \\
$r=5$ & 1.432 \\
\hline
\end{tabular}

Table 2: $h_{\max }$ for system (33) w.r.t. $r$

$A_{d}(\theta)=\sum_{i=0}^{\mu} A_{d i}$ with $A_{d i}=0, \forall i \geq 3$, we introduce therefore fictitious operators $\delta_{r+1}, \delta_{r+2}, \ldots$, which slightly improved results as shown in Table 2. Combining these two effects, the choice of the polynomial degree and the number of higher derivatives, we expect an improvement of our results. Hence, using $r=9, l=9$, we prove that system is stable for $h \leq 1.758$.

The second example is a second order distributed system of the form (2):

$$
\begin{aligned}
\dot{x}(t) & =\left[\begin{array}{cc}
0.2 & 0.01 \\
0 & -2
\end{array}\right] x(t) \\
& +\int_{-h}^{0}\left[\begin{array}{cc}
-1+0.3 \theta & 0.1 \\
0 & -0.1
\end{array}\right] x(t+\theta) d \theta
\end{aligned}
$$

Obviously this system is unstable for $h=0$. Furthermore, using the analytical method proposed by [2] and a gridding technic, this system is stable for $0.195 \leq$ $h \leq 1.71$. Using Theorem 4 over sliding windows, we obtain the following results summarized in Table 3. Finally, gathering all these intervals, we prove then that system (34) is stable $\forall h \in[0.20,1.3]$.

\begin{tabular}{|c|c|c|}
\hline Theorem 4, & $h_{\min }$ & $h_{\max }$ \\
\hline$l=1, r=1$ & 0.23 & 1 \\
$l=1, r=2$ & 0.21 & 1.2 \\
$l=1, r=3$ & 0.2 & 1.29 \\
\hline$l=2, r=1$ & 0.21 & 1 \\
$l=2, r=2$ & 0.20 & 1.2 \\
$l=2, r=3$ & 0.20 & 1.3 \\
\hline
\end{tabular}

Table 3: $h_{\min }, h_{\max }$ for system (34) w.r.t. $l$ and $r$ 


\section{Conclusion}

The paper is dedicated to the stability of distributed delay system. We assume that the delay kernel is a polynomial function, a wider class than usually used in the literature. Modelling this system as an interconnected feedback system allows to perform some new stability by using the quadratic framework. Future works include the extension of this technique to systems with mixed delays, neutral, pointwise and distributed delays. Another interesting work is the extension of this work to more general kernels. Approximating the kernel by a polynomial and modelling the related error by a bounded uncertainty seems to be a promising way.

\section{References}

[1] Y. Ariba, F. Gouaisbaut, and D. Peaucelle. Stability analysis of timevarying delay systems in quadratic separation framework. In International conference on mathematical problems in engineering, aerospace and sciences (ICNPAA'08), Genoa, Italy, 2008.

[2] D. Breda, S. Maset, and R. Vermiglio. Pseudospectral differencing methods for characteristic roots of delay differential equations. SIAM Journal on Scientific Computing, 27(2):482-495, 2005.

[3] W.-H. Chen and W.X. Zheng. Delay-dependent robust stabilization for uncertain neutral systems with distributed delays. Automatica, 43(1):95 $104,2007$.

[4] Y. Ebihara, D. Peaucelle, D. Arzelier, and T. Hagiwara. Robust performance analysis of linear time-invariant uncertain systems by taking higherorder time-derivatives of the states. In $44^{\text {th }}$ IEEE Conference on Decision and Control and the European Control Conference (CDC-ECC'05), Seville, Spain, December 2005.

[5] Y. A. Fiagbedzi and A. E Pearson. A multistage reduction technique for feedback stabilizing distributed time-lag systems,. Automatica, 23(3):311$326,1987$.

[6] E. Fridman and U Shaked. A descriptor system approach to $\mathcal{H}_{\infty}$ control of linear time-delay systems. IEEE Transactions on Automatic Control, 47:253-270, February 2002.

[7] E. Fridman and U Shaked. An improved stabilization method for linear time-delay systems. IEEE Transactions on Automatic Control, 47:19311937, November 2002.

[8] H. Gorecki, S. Fuksa, P. Grabowski, and A. Korytowski. Analysis and Synthesis of Time Delay Systems. John Wiley \& Sons, 1989.

[9] F. Gouaisbaut. Stability and stabilization of distributed time delay systems. In $44^{\text {th }}$ IEEE Conference on Decision and Control and the European Control Conference (CDC-ECC'05), Seville,Spain, 2005. 
[10] F. Gouaisbaut and D. Peaucelle. Robust stability of time-delay systems with interval delays. In $46^{\text {th }}$ IEEE Conference on Decision and Control (CDC'07), New Orleans, USA, December 2007.

[11] K. Gu, V. L. Kharitonov, and J. Chen. Stability of Time-Delay Systems. Birkhäuser Boston, 2003. Control engineering.

[12] Q.-L. Han. A descriptor system approach to robust stability of uncertain neutral systems with discrete and distributed delays. Automatica, 40(10):1791 - 1796, 2004.

[13] T. Iwasaki and S. Hara. Well-posedness of feedback systems: insights into exact robustnessanalysis and approximate computations. IEEE Transactions on Automatic Control, 43:619-630, May 1998.

[14] V. B. Kolmanovskii and A. Myshkis. Introduction to the Theory and Applications of Functional Differential Equations. Kluwer Academic Publishers, 1999.

[15] V. B. Kolmanovskii and J. P. Richard. Stability of some linear systems with delays. IEEE Transactions on Automatic Control, 44:984-989, May 1999.

[16] V. B. Kolmanovskii and L. E. Shaikhet. Control of systems with aftereffect. American Mathematical Society, 1996.

[17] V.B. Kolmanovskii, P.A. Tchangani, and J.P. Richard. Stability of linear systems with discrete-plus-distributed delay: application of some model transformations. In Conference on Mathematical Theory of Networks and Systems (MTNS'98), Padova, Italy, July 1998.

[18] X.-G. Li and X.-J. Zhu. Stability analysis of neutral systems with distributed delays. Automatica, 44(8):2197 - 2201, 2008.

[19] C.-I. Morarescu, S.-I. Niculescu, and Gu K. Stability crossing curves of shifted gamma-distributed delay systems. SIAM Journal on Applied Dynamical Systems, 6(2):475-793, 2007.

[20] U. Munz, J. M. Rieber, and F. Allgower. Robust stability of distributed delay systems. In 17th World IFAC Congress, Seoul, Korea, July 2008.

[21] S.I. Niculescu. Delay Effects on Stability. A Robust Control Approach, volume 269 of Lecture Notes in Control and Information Sciences. SpringerVerlag, Heildelberg, 2001.

[22] D. Peaucelle, D. Arzelier, D. Henrion, and F. Gouaisbaut. Quadratic separation for feedback connection of an uncertain matrix and an implicit linear transformation. Automatica, 43(5):795-804, 2007.

[23] P.A. Tchangani, Dambrine M., and Richard J.P. Robust stabilization of delay systems with discrete or distributed delayed control. In $37^{\text {th }}$ IEEE Conference on Decision and Control (CDC'98), pages 4051-4056, Tampa, Florida, December 1998. 